\title{
Factores que inciden en el financiamiento de los estudios Universitarios en Chile ${ }^{1}$
} SEBASTIÁN DONOSO*** E MARTIN SCHAFFERNICHT****

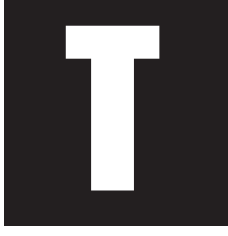

odo esfuerzo por mejorar el acceso igualitario a la educación universitaria enfrenta el desafío de contar con la suficiente disponibilidad de recursos para destinar a estudiar por parte de las familias y el Estado. Desde el año 1990 Chile ha hecho un esfuerzo significativo por elevar su gasto en educación y, particularmente en educación superior, proceso que ha sido exitoso pero fundamentalmente basado en un fuerte aporte familiar. Los resultados alcanzados muestran que esta situación está llegando a su punto de saturación. La sociedad chilena tendrá que debatir sobre los caminos que se adoptarán para enfrentar este problema, sea por la vía de un mercado

\footnotetext{
* Ingeniero Civil Industrial, Master en informática, Académico Facultad de Ciencias Empresariales Universidad de Talca, rschmal@utalca.cl.Chile.

** Economista, Ph. D. en Economía, Académico Facultad de Ciencias Empresariales Universidad deTalca rruiz@talca.cl.Chile.

***Educador, Dr. en Educación, Académico del Instituto de Investigación y Desarrollo Educacional de la Universidad de Talca, sdonoso@utalca.cl; Chile.

****Martin Schaffernicht, Dr, en Ciencias de Gestión, Académico Facultad de Ciencias Empresariales Universidad de Talca, martin@utalca.cl.. Chile.

1 Se agradecen los comentarios de Francisco Javier Gil, Pedro Henríquez, Alicia Leiva, Carlos Lorca, Patricio Ortúzar, Manuel Riesco y Carolina Velasco en el marco del Segundo Taller sobre Financiamiento de los Estudios Universitarios, realizado en Julio de 2005 en el Campus Santiago de la Universidad de Talca, Chile.
} 
regulado o por otros procesos, lo que implica revisar esquemas solidarios de financiamiento, que fortalezcan el principio de acceso igualitario.

El artículo se orienta a identificar los factores que inciden en la problemática señalada y, a partir de ello, sentar las bases para establecer el volumen de los recursos y sus opciones de provisión.

\section{Presentación}

En los últimos diez años en Chile se ha observado una aceleración en la tasa de matrícula de estudiantes universitarios tanto del sistema público como privado. Este crecimiento era predecible por varias razones. En primer lugar, desde hace varios años se ha implementado un conjunto de políticas y programas orientados a ampliar la cobertura de la enseñanza media y a mantener los estudiantes de ese nivel en el sistema educacional, con el fin de asegurar un mayor número de jóvenes egrese de ese nivel. Este objetivo se ha visto fortalecido por la norma constitucional aprobada en el año 2003 que establece la obligatoriedad de doce años de enseñanza, imponiendo el deber al Estado de proveer los recursos para cumplir este mandato.

En segundo lugar, el aumento en los ingresos de los hogares y la creciente importancia que las familias, particularmente de menores ingresos, otorgan a la educación, han incentivado a los jóvenes a considerar la continuación de estudios en instituciones de educación superior. Materializar esta aspiración, que hasta los primeros años de la década de los noventa se percibía como lejana, es hoy viable para un creciente número de familias pertenecientes a los primeros quintiles socioeconómico (los más pobres).

En tercer lugar, la clara concepción de mercado que impera en todo el sector educación, han generado incentivos para expandir la oferta de cupos y nuevas carreras, lo que a su vez contribuye a lograr que esta demanda potencial encuentre los canales para su realización, conducta que se ve 
fortalecida por el alto retorno privado que aún muestra la formación universitaria (Sapelli, 2002).

La expansión de la cobertura y las activas políticas educacionales de los niveles anteriores, particularmente de la educación media, han reducido sustantivamente las brechas que se observan entre los distintos quintiles de ingreso. Aún cuando la demanda en los sectores de mayores ingresos no se ha saturado, el nivel de cobertura que se observa en los quintiles superiores es similar a los promedios que se registra en los países más avanzados. En consecuencia, la expansión de la demanda en educación superior tiende a concentrarse en jóvenes que provienen de los hogares de menores ingresos.

En síntesis, el conjunto de factores mencionados anteriormente, inducen fuertemente a los sectores sociales que han estado excluidos a incorporarse a la educación superior (Brunner y Elaccqua, 2003). En la medida que los jóvenes sienten que han logrado pasar las barreras del ámbito estrictamente educacional y que las dificultades a superar se sitúan en el campo financiero, es esperable que ello se traduzca en una creciente demanda por recursos adicionales para que el sistema de educación superior pueda acogerles.

Por su parte, en el ámbito del Estado y de los agentes políticos que compiten por el gobierno para implementar sus políticas, se ha instalado la convicción que para avanzar hacia mayores niveles de desarrollo, el país debe acrecentar sus esfuerzos en inversión en capital humano, y en iniciativas orientadas a generar condiciones para la innovación y de desarrollo científico y tecnológico, donde es fundamental el componente de una masa laboral más calificada. En consecuencia, dado que la principal fuente de incremento potencial de estudiantes proviene de hogares pertenecientes al tercer quintil, tendencia que probablemente continúe hacia los quintiles inferiores, es posible identificar a lo menos tres desafíos que debe enfrentar el Estado: por una parte, diseñar nuevos instrumentos de financiamiento; 
segundo, ampliar la disponibilidad de recursos para financiar los estudios de educación superior , y finalmente, diseñar mecanismos de asignación que optimicen su impacto.

El tema del financiamiento puede ser abordado desde diversas perspectivas. En el caso chileno se han privilegiado opciones de mercado postergando la implementación de mecanismos alternativos como una ampliación de las becas, aranceles diferenciados y otros. En la actualidad, no se dispone de un modelo comprehensivo que incorpore un conjunto de factores relevantes y reglas que permitirían determinar el nivel de recursos que sería deseable dedicar al financiamiento de los estudiantes de educación superior. Hasta ahora el debate se ha centrado en opciones de mercado, fundamentalmente a través de líneas de crédito para estudiantes, que a la fecha han operado con los principios de un fondo rotatorio con características muy particulares -las que se analizan más adelante.

El propósito de este trabajo es formular una propuesta que se circunscribe a resolver para el sistema universitario, el segundo desafío enunciado: identificar los factores y sus relaciones que inciden en la oferta y demanda de recursos que permitan financiar los estudios universitarios.

Si bien el sistema de educación superior chileno contempla diversos niveles (Centros de Formación Técnica, Institutos Profesionales y Universidades), en este trabajo se ha optado por abordar sólo el sistema universitario, dado que es en este nivel donde actualmente se aplica el modelo de financiamiento vía crédito fiscal y por su peso dentro del financiamiento total requerido para el conjunto del sistema. Posteriormente este modelo puede ser replicado en los otros niveles.

El trabajo se organiza en tres secciones: la primera, describe el sistema universitario chileno. La siguiente sección trata el tema del financiamiento de los estudiantes. Finalmente, se presenta el modelo conceptual (causal) del sistema. 


\section{Algunos antecedentes del sistema universitario chileno}

El actual sistema universitario chileno es de carácter mixto. El número de estudiantes se estima en 350.000 (CSE, 2004), de los cuales aproximadamente 215.000 están matriculados en las universidades adscritas al Consejo de Rectores (identificadas como universidades públicas). ${ }^{2}$ El resto corresponde a universidades de origen y financiamiento privado, denominadas "extra-consejo".

El Estado es responsable de una parte importante del financiamiento de las universidades que pertenecen al Consejo de Rectores y, consecuen-temente no puede eludir su compromiso con el uso y los resultados que se generan. Considerado en su conjunto los estudiantes de pregrado de las universidades públicas y privadas representan una inversión anual en capital humano cercano a US\$ 800 millones. ${ }^{3}$ Es altamente probable que este gasto anual en inversión tienda a aumentar no sólo por la incorporación de nuevos estudiantes, sino por la necesidad de mejorar la calidad de la formación.

Desde hace algunos el sistema de financiamiento de los estudios universitarios de pregrado basado en el crédito universitario (solidario) evidencia crecientes signos de agotamiento, aumentando la brecha entre la suma total del monto solicitado y el disponible. Esta situación es exacerbada por los mecanismos de recuperación del crédito, que dada la forma como fue definido no garantiza la disponibilidad futura de fondos que se requieren para cubrir las nuevas demandas. ${ }^{4}$

2 Conformadas por 25 planteles, dieciséis estatales, seis universidades católicas y tres corporaciones privadas.

3 Asumiendo un arancel promedio anual de $\$ 1,500,000$, una tasa de cambio de 1 US $\$=\$ 650$ y una matrícula universitaria de 350,000 alumnos.

4 El crédito implica un plazo máximo de pago de 15 años, al término del cual se extingue la deuda impaga cualquiera sea su monto. El pago que realiza el ex alumno es contingente y representa hasta un $5 \%$ de los ingresos. Hasta ahora ha operado como un sistema subsidiado en aproximadamente un $50 \%$. 
En razón de lo señalado, esta situación se ha tornado crítica, siendo uno de los principales focos de discordia entre el gobierno, las autoridades universitarias y los estudiantes, problemática que a la fecha no ha sido resuelta satisfactoriamente.

\section{La discusión central acerca del financiamiento de los estudiantes}

El debate que se ha generado en torno al actual sistema de financiamiento de los estudios universitarios en Chile ha permitido revelar el carácter estratégico del mismo. Desde esta perspectiva, el actual formato de financiamiento requiere un proceso de reingeniería que incorpore al menos tres factores claves para definir el problema y, consecuentemente, insertarlo en una completa revisión del modelo de desarrollo del sector.

Primero, el progresivo aumento del número de estudiantes universitarios que se espera para los próximos años y la condición socioeconómica de los mismos se traducirá inevitablemente en crecientes demandas por un mayor financiamiento público, presiones que de no tener su cauce adecuado podrán dar paso a conflictos sociales de magnitud. ${ }^{5}$ En segundo lugar, y considerando las externalidades positivas que representa para el país un avance en la tasa de profesionalización de la masa laboral, requiere diseñar e implementar un sistema de financiamiento sostenible en el tiempo. Por último, está la responsabilidad del Estado por asegurar un desarrollo del sistema universitario compatible con las aspiraciones estratégicas del país, y que permita a toda la población avanzar hacia estadios superiores de bienestar contribuyendo simultáneamente a construir una sociedad inclusiva y con mayor cohesión social.

5 Situación que puede reforzarse dado que a partir del año 2004 el Estado chileno decretó la enseñanza media como nivel educativo obligatorio, impulsando por esta vía la escolarización de toda la población y, en razón de ello es previsible una mayor demanda por educación terciaria. 
El colapso del actual sistema de financiamiento de estudios universitarios es más que un fenómeno perceptual. Anualmente los montos de incremento real del fondo de crédito solidario se ven superados por su demanda. ${ }^{6}$ Los significativos incrementos del Fondo para becas y créditos estudiantiles (que representan algo más del 30\% de los recursos destinados a educación superior) son y han sido insuficientes para una adecuada satisfacción de la demanda (Larrañaga, 2002: 7-8). No obstante el crecimiento de los recursos fiscales señalados es menor que la tasa bajo la cual aumenta la matrícula universitaria, así como su costo. En los últimos 20 años la matrícula en las universidades del Consejo más que se duplicó, sin embargo la tasa de graduación no ha seguido ese patrón (CSE, 2004). Paralelamente, por primera vez desde 1981, las universidades extra-consejo ofrecieron el año 2004 más matrículas de primer año que las del Consejo (CSE, 2004).

Si bien la nueva ley podría contribuir en parte a solucionar este fenómeno, es posible que esta no resuelva el problema sino cuenta con los fondos requeridos para satisfacer adecuadamente la progresión de la demanda, tanto en los remanentes actuales como en las demandas futuras. Lo que puede tender a agravarse porque el valor de los aranceles está siendo fijado con independencia de la disponibilidad de crédito.

Complementariamente, el valor de los aranceles se ha incrementado por sobre el índice de precios al consumidor, y aún cuando los precios que rigen en las universidades privadas son en promedio un 23\% más altos que los valores que rigen en las públicas, la tendencia que se observa es que ambos tienden a igualarse. En cierto modo podría afirmarse que el precio de seguir estudios universitarios está tendiendo a un "precio de equilibrio de mercado" sin mayores limitaciones que las que reporta el comportamiento

6 Véase al respecto el presupuesto inicial del Ministerio de Educación para la educación superior, su reitemización y el presupuesto finalmente ejecutado por años (MINEDUC 1999, 2000, 2001, 2001, 2002). 
de la demanda y la oferta, con un Estado que a lo más cumple un rol de "observador". Estos aumentos, en especial en las universidades del Consejo, se han basado esencialmente en la demanda del mercado de educación superior, sin considerar la evolución de los fondos disponibles para el crédito universitario ni la capacidad de recuperación de los créditos concedidos. Ciertamente el incremento de la oferta de vacantes ha sido uno de los factores claves que ha impulsado la demanda además del aumento en la escolaridad promedio de la población a una cifra superior a los diez años (INE, 2004), la cual se elevará en los próximos años con la nueva normativa de escolaridad media obligatoria. A esto se agrega el incremento significativo (más de un 5\%) en la cobertura, tasa de éxito oportuno y tasa de éxito total de estudiantes de la enseñanza media (MINEDUC, 2002). Estos factores harán crecer el número de jóvenes demandantes de educación terciaria en una cifra superior al crecimiento vegetativo de la población de estudiantes. A ello han de sumarse los que tienen sobreedad (adultos que quieren estudiar). Es claro que en nuestro país la demanda por educación universitaria continuará incrementándose en los próximos años, estimándose que sólo después del año 2010 se estabilizará, entre otros factores por el estancamiento de la tasa de crecimiento de la población (CEPAL/CELADE, 2000), la saturación de los mercados laborales, etc., alcanzando el sistema universitario una masa de estudiantes cercana a los 800.000 alumnos de pregrado.

Como se observa en la Figura $N^{\circ} 1$, entre 1990 y 2003, la cobertura de educación superior creció desde 16,0\% a 37,5\% (2,3 veces); los mayores incrementos se producen en la población de los quintiles de mayores ingresos. No obstante, la cobertura entre los jóvenes del $40 \%$ de los hogares de menores ingresos se triplica en el periodo, pasando en el caso del primer quintil de $4,4 \%$ a $14,5 \%$, y de $7,8 \%$ a $21,2 \%$, en el segundo. Por lo tanto en el próximo quinquenio, habrá un mayor requerimiento de financiamiento de los estudios universitarios por parte de una población que a su vez demandará una mayor cobertura de crédito. 
Sociologias, Porto Alegre, ano 9, no 17, jan./jun. 2007, p. 316-339

Cobertura de educación superior por quintil de ingreso autónomo per capita del hogar, 1990 y 2003

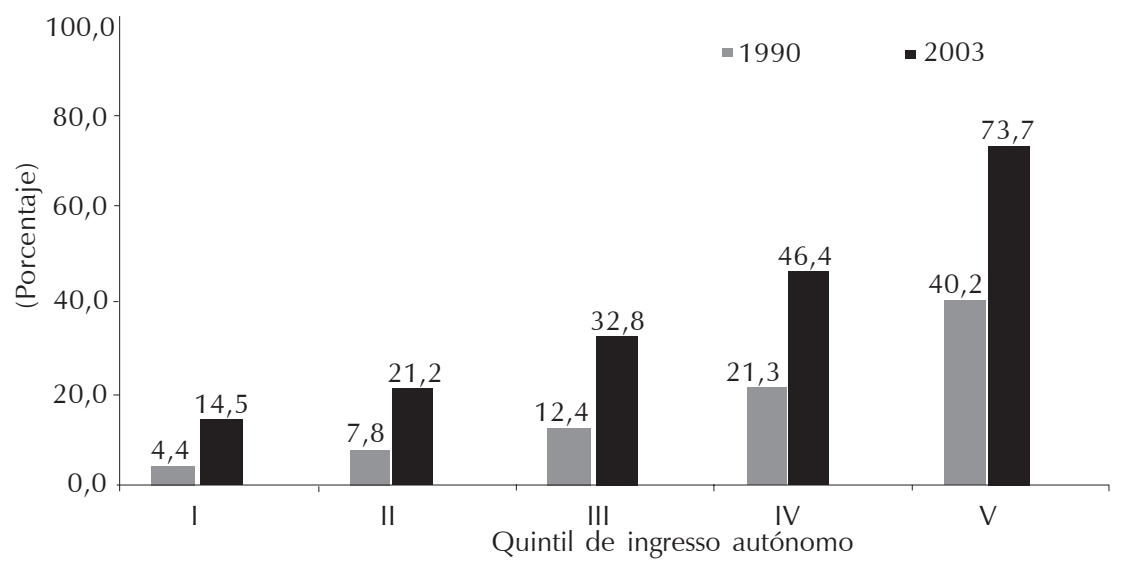

En la actualidad solo pueden acceder al fondo solidario de crédito estudiantil los alumnos de las universidades del Consejo, excluyéndose los estudiantes de universidades extra-consejo. Los argumentos para mantener inalterable esta situación se debilitan con el tiempo, más aún cuando la orientación del pregrado de muchas universidades públicas es similar a las privadas. En consecuencia se está marginando del acceso al sistema de crédito solidario a más del $50 \%$ de los alumnos matriculados en universidades. Por tanto, sería razonable esperar mayores requerimientos de financiamiento público para los estudios universitarios dada la creciente matrícula en universidades privadas, cuyos alumnos, que provienen en forma creciente de hogares de los quintiles inferiores, no tienen hoy acceso al crédito solidario ni a las becas que ofrece el Estado.

La magnitud del fondo solidario de cada universidad del Consejo obedece en lo sustancial a razones históricas, sin mayor relación con la composición socioeconómica del alumnado ni la evolución de su matrícula. 
De allí que existan grandes variaciones en el nivel de ingresos mensuales per cápita de quienes acceden al crédito, dependiendo de la universidad que se trate. En consecuencia, se observa un desigual acceso de los alumnos al crédito según la universidad en la cual estudien.

No parece razonable que universidades del Consejo que concentren mayor proporción de alumnos provenientes de familias con altos ingresos perciban montos que les permita asignar crédito a quienes de matricularse en otras universidades no lo tendrían. Para subsanar lo expuesto, el grueso de las universidades que exhiben insuficientes fondos para cubrir las demandas de crédito, enfrentadas situaciones de alta conflictividad -paros y movilizaciones, finalmente destinan recursos propios, de fuentes que tenían otros objetivos con el consiguiente impacto en inversiones, investigación y docencia.

La recuperación de los créditos no está funcionando apropiadamente -entre otros motivos- por la baja capacidad de cobranza, lo que se traduce en un menor retorno de los recursos previstos originalmente para este sistema. Aún cuando la recuperación fuese del 100\%, la recaudación sería insuficiente para satisfacer la demanda crediticia tanto por la evolución de esta última, como por las características de los créditos asignados. Si bien desde el comienzo el crédito fiscal incorporaba un subsidio implícito, éste ha aumentado por la concomitancia entre una tasa de interés muy por debajo de la del mercado 7 (2\%), un período de gracia de 2 años, la limitación de pagar hasta el $5 \%$ de los ingresos que se obtienen en la vida profesional, y un plazo máximo de 15 años para amortizar el crédito, después del cual se condona el saldo impago.

Lo anterior ha dado origen a una deuda acumulada cercana a US $\$ 1.200$ millones, dado que el sistema en su conjunto no alcanza a recuperar

7 La nueva ley de financiamiento que se propone este año (2004) mantiene la tasa de interés, amplia el plazo de recuperación a 20 años, e igualmente sostiene la recuperación asociada a contingencia. 
anualmente el $50 \%$ de lo que corresponde. ${ }^{8}$ El resultado deriva en universidades acosadas por demandas incapaces de satisfacer.

Chile es un país que exhibe una alta desigualdad socioeconómica, la que se expresa, entre otras características, en que sólo un 20\% de quienes postulan a la educación superior provienen de familias con capacidad para financiar sus estudios. Para el perfil de ingreso per cápita que tiene el país, del orden de los US $\$ 5.500$ pero con una estructura altamente regresiva, el costo anual de los estudios universitarios es en extremo oneroso, pudiendo variar entre los US \$1.200 y US \$3.500 por alumno según la universidad y carrera que se trate. ${ }^{9}$ Para ilustrar este punto bastaría señalar que para la prueba de selección universitaria del año 2005 se inscribieron casi 170.000 estudiantes, de los cuales más del 50\% declaró ingresos familiares no superiores a los US\$ 500 mensuales.

La importancia del capital humano en la innovación y generación de ingresos está presente en todas las teorías recientes de crecimiento y desarrollo económico. Dicho aporte se manifiesta primordialmente a través del aumento en la productividad, al facilitar la adopción de tecnologías y procesos productivos más sofisticados y flexibles. La contribución del capital humano al crecimiento se encuentra respaldada por una extensa investigación empírica (véase Las metas del milenio de Naciones Unidas) que muestra que buena parte del crecimiento económico de los países es explicado por la Productividad Total de Factores (PTF) y que un elemento clave para el crecimiento de ésta corresponde a la calidad de la educación. A este respecto se ha argumentado que si Chile mejorase la calidad de la educación, la productividad aumentaría a medida que las nuevas generaciones se integren a la fuerza de trabajo. Empíricamente se ha estimado que un aumento en la calidad de la educación consecuente con el logro de resultados promedio

8 En el presente año se recuperaron poco menos de 50 millones de dólares ( $M M$ \$31.000) 9 A una tasa de cambio US $\$ 1=\$ 650$. 
para el nivel de ingreso del país llevaría a aumentos de hasta 0,7 puntos porcentuales en la PTF.

Para enfrentar el desafío de la economía del conocimiento es relevante la calidad, cobertura y equidad en acceso a la educación superior. En este sentido, actualmente Chile presenta una cobertura de $37,5 \%$ cifra que es significativamente inferior al 63,6\% que exhiben países considerados innovadores como Suecia, Corea del Sur, Finlandia y Suecia (Brunner y Elacqua, 2003).

Los antecedentes anteriores permiten dimensionar el desafío que enfrenta la sociedad chilena en que, por una parte le asigna un creciente valor a mejorar la calidad y cobertura de la educación en todos sus niveles y, al mismo tiempo, requiere incorporar a un segmento de la población que hasta ahora aparece excluido del sistema de educación superior.

Estadísticas del Banco Mundial indican que en Chile sólo un 10,2\% de la fuerza laboral es técnico-profesional, en contraste con los países innovadores, donde esta cifra se eleva al 29,2\%. Pero la principal fuente de preocupación respecto a la educación superior es la existencia de diferenciales de rentabilidad excesivamente altos. Éstos no sólo ayudan a explicar la desigualdad distributiva, sino que son indicativos de distorsiones que amenazan con prolongar dicha desigualdad en el tiempo, perjudicando las posibilidades de crecimiento futuro del país. Lo anterior tiene que ver con la falta de equidad en el acceso a la educación superior. Aunque desde 1990 la proporción de jóvenes del 40\% más pobre de la población que accede a la educación superior prácticamente se triplicó — pasó de 4,4\% a $14,5 \%$ en el primer quintil de ingresos y de $7,8 \%$ a $21,2 \%$ en el segundo, la brecha de acceso entre este grupo y el $20 \%$ más rico no se ha reducido. A la luz de la alta rentabilidad privada de la educación superior, esto significa que el desarrollo de este sistema no está ayudando a generar mayor equidad para el futuro. 


\section{Caracterización del modelo de análisis propuesto}

El modelo diseñado busca precisar el impacto financiero en distintos escenarios representados por los cambios en las variables claves del problema en consideración. Lo más significativo del mismo es que incorpora consideraciones respecto de la intemporalidad de las relaciones entre variables que hasta a la fecha no habían sido consideradas, a saber: la tasa de profesionalización, el aporte de éstos a los ingresos económicos y a la generación de ingresos futuros para el Estado a través de la tributación, etc.

En lo operacional, para diseñar el modelo se han introducido los siguientes supuestos:

- Las variables involucradas en el modelo y sus relaciones causales son comunes a cada uno de los quintiles socioeconómicos de la población.

- Los alumnos seleccionados para ingresar a las universidades reúnen las capacidades académicas para culminar exitosamente sus estudios.

- Cada carrera tiene un arancel anual fijado autónomamente por cada universidad con independencia de la disponibilidad de crédito y de los ingresos familiares.

- Para los efectos del modelo, en una primera aproximación, se considera un arancel único o promedio. Este arancel es el valor que debe pagar anualmente un estudiante por los servicios docentes que la universidad se compromete a entregar durante el año.

- El nivel de disponibilidad de recursos económicos es función del quintil al que pertenece la familia del estudiante.

- El modelo mantiene el actual sistema de financiamiento universitario, basado en el pago de aranceles para los estudiantes del pregrado. Por lo tanto, según la situación socioeconómica del grupo familiar del estudiante, éste puede acceder a distintos fondos de crédito universitario. 
La figura $N^{\circ} 2$ expone el diagrama de ciclos (bucle) causal (Sterman, 2000), donde los nodos representan variables de distintas magnitudes y las flechas una relación de causalidad positiva (+) o negativa (-) dependiendo de la dirección de la variación de la variable afectada ante un cambio en la variable causante.

La figura ilustra los factores relevantes para determinar los fondos destinados al crédito universitario. El monto solicitado cada año se explica por las necesidades de dos poblaciones: la de los actuales estudiantes y la de los nuevos, que aparecen en el sistema como "nueva demanda". Desde la primera postulación hasta el momento de convertirse en profesionales, los estudiantes solicitan los créditos que requieren.

Al titularse pasan a ser profesionales y como tales, se les presume un ingreso sustancialmente superior al que podrían haber esperado obtener sin haberse titulado. Después del período de dos años de gracia, empiezan a rembolsar en base a una tasa, aportando así al monto disponible para nuevos créditos.

Esta figura muestra que a mayor devolución del crédito (reembolso), sería mayor la disponibilidad del fondo. Sin embargo, aún cuando el reembolso fuera de $100 \%$, y la demanda no aumentara y el valor de los aranceles se mantuviera constante, este fondo difícilmente podría ser autosustentable en virtud del componente de subsidio que contiene. Pese a que en los últimos años se ha puesto especial énfasis en mejorar los sistemas de cobranza, esta política no es suficiente para la sustentabilidad del Fondo.

Parte del crecimiento de la demanda por crédito puede ser inducida por un mayor nivel del fondo: en la medida que los jóvenes sepan de una mayor disponibilidad de recursos aumentarán la postulación a las universidades. Otra parte puede provenir del aumento de los aranceles de las universidades, lo que incide en una mayor necesidad de recursos para crédito.

En consecuencia, bajo las condiciones actuales (crecimiento de demanda, aumento de los aranceles y recuperación inferior a 100\%), parece 


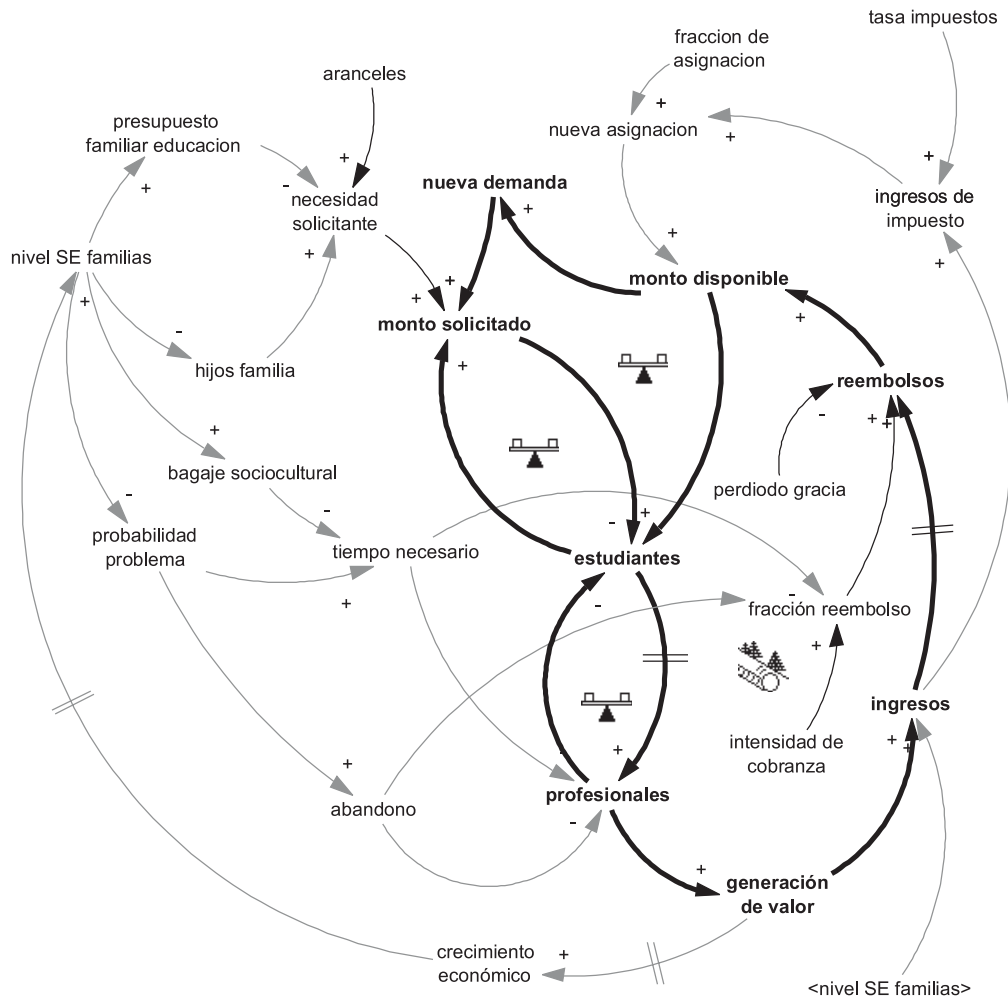

Figura 2 - La intensidad de la cobranza y el período de gracia regulan la recuperación, y en ausencia de nueva demanda podrían estabilizar el sistema. Sin embargo, una mayor disponibilidad de fondos tiende a aumentar la cantidad demandada. Por su parte cualquier aumento de los aranceles implica incrementar la necesidad de crédito de cada solicitante. Por tanto ambos aspectos inciden sobre el monto solicitado. Se observa que la incorporación de recursos adicionales al Fondo tiene un doble efecto: aumenta las posibilidades de financiar el monto solicitado (auto-estabilización), y también aumenta la demanda, lo que disminuye la posibilidad de financiarla totalmente. Solamente cuando se agota la fuente de nueva demanda deja que actuar el segundo bucle (negativo). En este sentido, la asignación adicional de recursos es un incentivo para estudiar. 
impensable que el crédito universitario funcione adecuadamente sin asignaciones anuales adicionales. Surge entonces la pregunta: ¿cuánto se necesita? o bien ¿̇cómo determinar el monto de la asignación adicional que se requiere?

Para responder estas interrogantes es necesario considerar los siguientes factores:

a) el posible aumento de ingresos del Estado vía impuestos;

b) la posible disminución de la necesidad de crédito de los jóvenes;

c) los riesgos de un flujo insuficiente de recursos.

Las siguientes secciones ilustran cada uno de estos aspectos.

El posible aumento de ingresos del Estado por los impuestos

Los estudios universitarios dan origen a mayores ingresos futuros por parte de quienes se matriculan en las universidades, razón por la cual como profesionales pagarán más impuestos de lo que habrían pagado sin estudiar.

Por tanto, por haber otorgado recursos para crédito el Estado obtendrá ingresos adicionales. De allí que el crédito debe concebirse como una inversión que genera ingresos futuros. Incluso es posible pensar que parte de estos ingresos adicionales puedan ser destinados a aumentar el monto disponible. En este caso, la nueva asignación permite incrementar el número de estudiantes que algunos años más tarde pagarán más impuestos de los que habrían pagado sin estudiar. Simultáneamente actúa el bucle de la incitación de nueva demanda, la que se mantendrá activada hasta agotarse la nueva demanda potencial.

Actualmente se desconocen la magnitud del ingreso adicional de impuestos en relación con la asignación de recursos adicionales y su distribución en el tiempo. Sin embargo, este efecto existe y por lo tanto es parte fundamental del modelo para determinar el monto de una nueva asignación. 


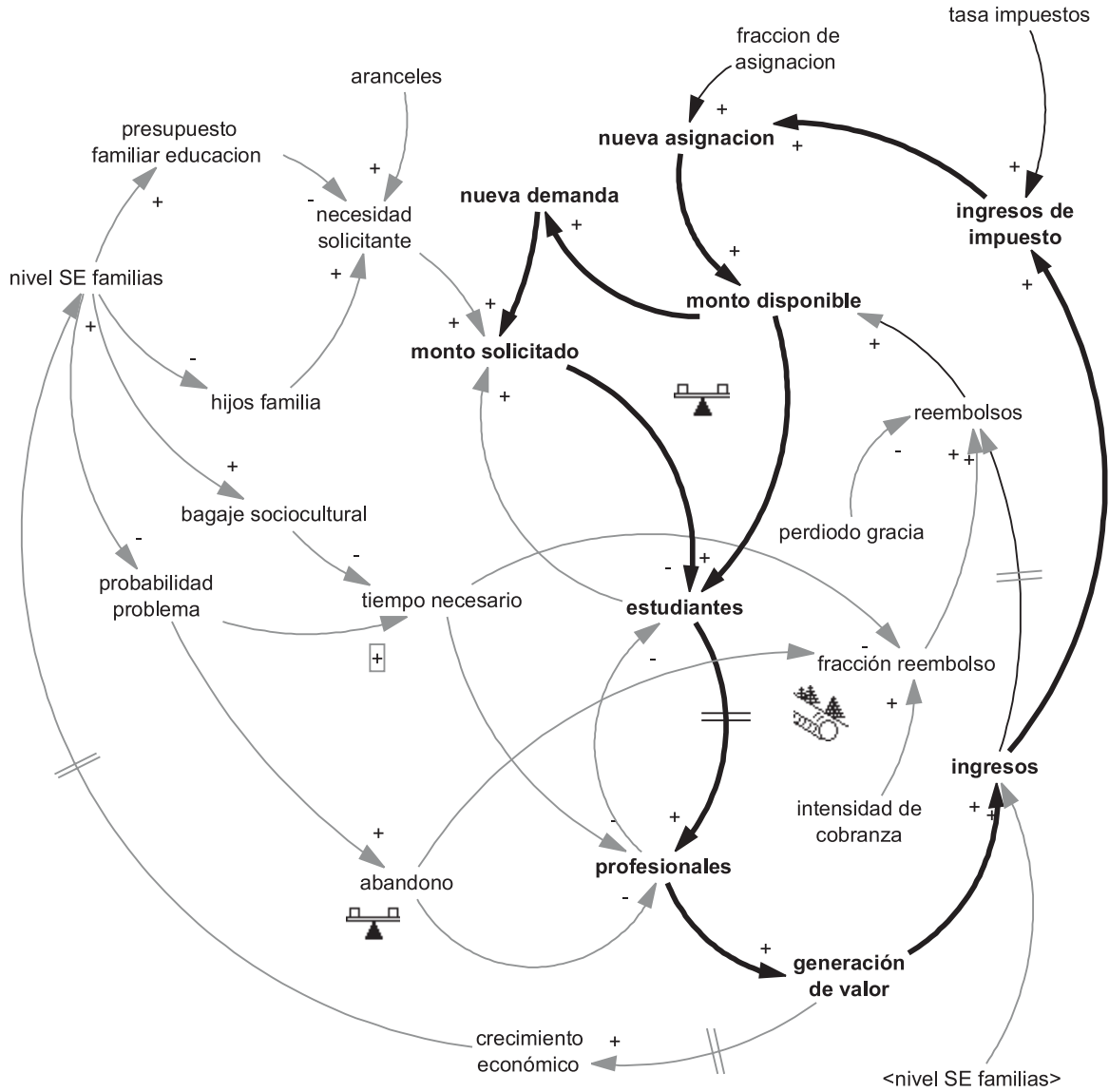

Figura 3 - Ingresos adicionales vía impuesto por el mayor ingreso de los nuevos profesionales. 
La posible disminución de la necesidad de crédito de los jóvenes

En general la Teoría del Capital Humano sostiene que los estudios universitarios tienen incidencia significativa en la movilidad social ascendente. Al mismo tiempo se observa que la cobertura de estudios universitarios de los quintiles socioeconómicos inferiores es reducida, en razón de ello se espera que el grueso del crecimiento de la demanda provenga de estos quintiles. Esta situación implicará aumentar la proporción de estudiantes cuyos padres -por lo general- no son profesionales. Por lo tanto es de esperar que en las décadas siguientes, cuando los hijos de estos estudiantes estén en edad para ingresar a la universidad, la familia tenga un nivel socioeconómico más alto, por lo que su necesidad de crédito será inferior a la que tuvieron sus padres.

Esto significa que en el lapso de una generación una menor proporción de estudiantes demandará crédito para financiar los aranceles. Sin embargo, esa menor demanda no considera los costos de alimentación, alojamiento y otros, cuyo financiamiento también puede ser objeto de demanda, ni tampoco se conoce la magnitud relativa del efecto intergeneracional.

No obstante estas dificultades, queda en evidencia que el efecto existe, y que debería considerarse en el diseño de una política para establecer el volumen deseado de las asignaciones.

Los grupos de ciclos (bucles) de retroalimentación tienen un comportamiento amplificador, que puede darse bajo dos modalidades: virtuosa o positiva por los mayores ingresos profesionales y la generación de valor para quienes contratan sus servicios; y la viciosa o negativa por la insuficiencia del crédito asignado que no permite satisfacer cabalmente las necesidades económico-financieras, con consecuencias en el rendimiento académico que retrasan el egreso de la universidad.

La modalidad viciosa o negativa es la que probablemente más pesa en la actualidad; si en un año el crédito no es suficiente para quienes lo necesitan, se pierde el efecto descrito para el futuro, el que no es factible de recuperar, quedando como una hipoteca que no logra cancelarse. 


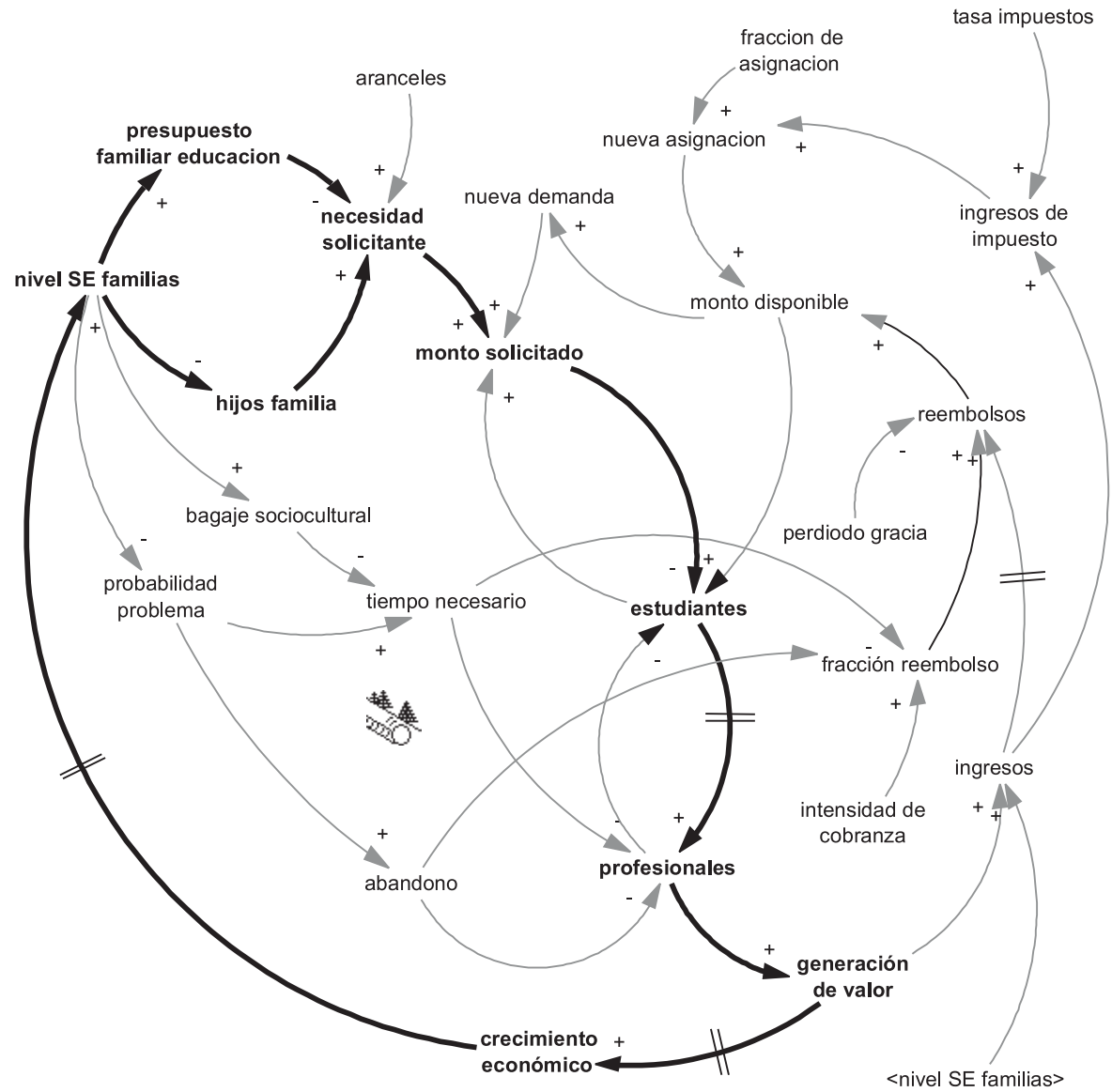

Figura 4 - Los hijos de los actuales estudiantes necesitarán menos crédito. 
Los riesgos de un flujo insuficiente de recursos

Existen diversas vías por las cuales la insuficiencia de fondos puede afectar al sistema. Una de ellas proviene del hecho que la mayoría de los nuevos estudiantes, al pertenecer a los niveles socioeconómicos más bajos, serán más vulnerables ante las reducciones de crédito. La otra es que esta nueva población de estudiantes, producto de su déficit de capital social, cultural etc. demorará en promedio más tiempo para terminar sus estudios.

El efecto de lo señalado será la postergación del inicio de su desempeño profesional y elemento activo en los dos grupos de ciclos (bucles) presentados. Esta postergación puede darse en forma de retiros temporales (por ejemplo, para trabajar) o retrasos en el pago de aranceles (lo cual provoca que la institución no entregue el título profesional). En ambos casos habrá menos profesionales de lo que habría sido posible; en consecuencia, no se obtiene todo el potencial de ingreso adicional de impuestos, ni toda la disminución de necesidades, reforzándose el riego de un flujo insuficiente de recursos.

Esto demuestra que a lo menos una parte de los problemas del sistema de crédito universitario son de carácter endógeno, excluyendo los hábitos de no pago o los generados por débiles sistemas de cobranza. De lo visto se desprende que insuficientes asignaciones adicionales tienen una gran capacidad para generar problemas de atraso y abandono con consecuencias que no pueden ser corregibles solo por una cobranza más estricta. 


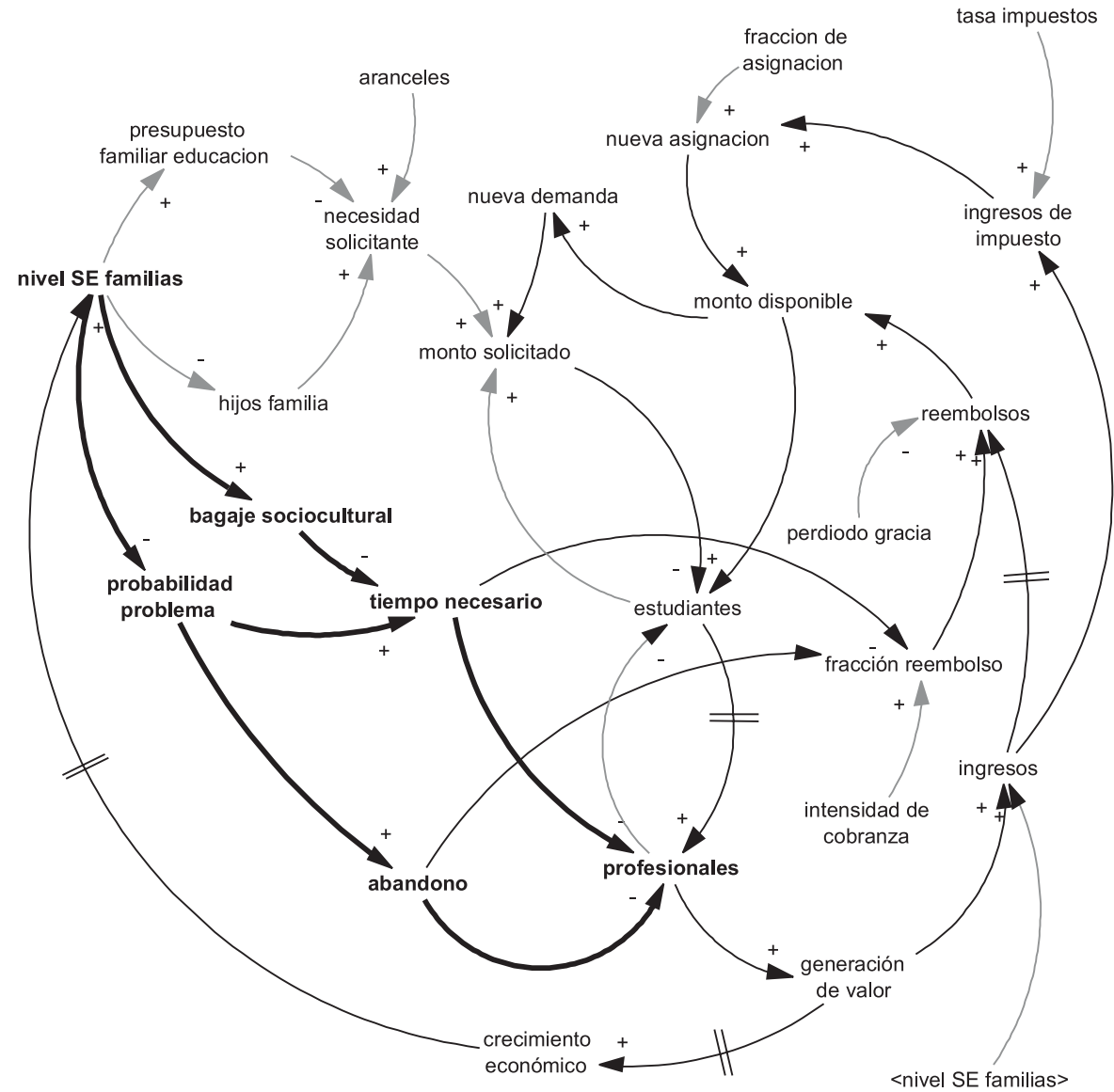

Figura 5 - Cada vez que un estudiante no logra obtener su título o se atrasa, hay menos profesionales en el mercado. Esto disminuye la recuperación, la recaudación de impuestos y el crecimiento que iba a ayudar a disminuir la necesidad. 


\section{Consideraciones finales}

El artículo elabora una propuesta relativa a los factores que deben considerarse en la determinación del monto de crédito deseable para el sistema universitario chileno. Se presenta un modelo causal (conceptual) que muestra múltiples bucles de retroalimentación.

El modelo propuesto permite representar la existencia de dos fuerzas que actúan en direcciones opuestas. Una en sentido positivo y la otra negativo. La positiva, proviene del aumento de la tasa de profesionales en la población activa, lo que se traduce -por un lado- en un aumento de los ingresos por impuestos y por otro, en la progresiva reducción de la necesidad de crédito. Ambas son efectos de mediano a largo plazo, que se retroalimentan y por tanto tienden a la auto-amplificación.

La negativa, se deriva de la creciente proporción de estudiantes que proviene de los quintiles más vulnerables. Cuando la disponibilidad de crédito per cápita no satisface los requerimientos de los estudiantes, aumenta la probabilidad que éstos abandonen o prolonguen sus estudios por factores económicos. En consecuencia se incrementa la escasez relativa del monto disponible, lo que tiende a postergar y reducir la incorporación de nuevos recursos al fondo.

El modelo asume que todos los postulantes que se incorporan como estudiantes poseen las competencias mínimas para desempeñarse satisfactoriamente en la universidad. Sin embargo, hay antecedentes que indican que el fracaso también tiene raíces académicas, en consecuencia no se puede atribuir el retraso o la deserción sólo a la variable socioeconómica, aunque ésta sigue siendo la más significativa. Al mismo tiempo se argumenta que las competencias académicas tiene fuertes nexos con la situación socioeconómica de la población, estableciéndose un círculo vicioso entre estos factores (González, 2005). 
El sistema del crédito universitario es una materia compleja, lo que no impide que sea representada ni revelada su estructura y comportamiento en este modelo. A partir de éste es posible diseñar una política de crédito universitario basada en los factores señalados. El trabajo futuro consiste en determinar el nivel de recursos deseable incorporando el factor intergeneracional y la fuerza con que inciden cada una de las variables incluidas en el modelo.

\section{Referencias}

BRAVO, D. y CONTRERAS, D. Competencias básicas de la población adulta. CORFO, Universidad de Chile, Ministerio de Economía. Santiago, Chile, 2001.

BRUNNER, J.J. y ELACCQUA, G. Capital humano en Chile. Universidad Adolfo Ibáñez, Santiago, Chile, 2003.

CASEN. Resultados iniciales Encuesta Nacional de Caracterización Socioeconómica. Ministerio de Planificación Nacional. Santiago. www.mideplan/casen. 2004.

CEPAL/CELADE. Boletín demográfico, no 66. División de Población, Santiago. 2000.

CSE. Indicadores Generales de Educación Superior. Consejo Superior de Educación, Chile. www.cse.cl. 2004.

GONZÁLEZ, P. La igualdad educativa, el financiamiento vía subvenciones y la administración privada de la educación. UNESCO. Políticas Educativas y Equidad. Santiago de Chile, Pp. 247 -270, 2005.

INE. Resultados Censo Nacional de Población y Vivienda 2002. Instituto nacional de Estadísticas, Chile. www.ine.cl. 2004.

MINEDUC. Anuario Estadístico. Departamento de Estudios y Desarrollo de la División de Planificación y Presupuesto del Ministerio de Educación, 1999.

MINEDUC. Anuario Estadístico. Departamento de Estudios y Desarrollo de la División de Planificación y Presupuesto del Ministerio de Educación. 2000. 
MINEDUC. Anuario Estadístico. Departamento de Estudios y Desarrollo de la División de Planificación y Presupuesto del Ministerio de Educación. 2001.

MINEDUC. Anuario Estadístico. Departamento de Estudios y Desarrollo de la División de Planificación y Presupuesto del Ministerio de Educación. 2002.

MINEDUC. Indicadores de la Educación Chile 2002. Departamento de Estudios y Desarrollo de la División de Planificación y Presupuesto del Ministerio de Educación. 2002.

SAPELLI, C. Tasas de retorno de la educación en Chile. 1990-1998. www.edec.Cl/ fceaecono. 2002.

STERMAN, 2000. Business Dynamics. 2000. John Wiley.

STIGLITZ, J. La economía del sector público. Antoni Bosch, editor. España. 2002.

\section{Resumen}

El artículo da cuenta de los principales factores que inciden en el financiamiento de los estudios universitarios en Chile, exponiéndose los fundamentos y relaciones de un modelo para estimar el volumen de los recursos comprometidos y sus opciones de provisión. En las últimas dos década el mercado universitario ha duplicando su masa de estudiantes fenómeno que continuará por esta senda, implicando serias presiones sobre el financiamiento público, en un sistema que ya tiene una elevada participación financiera privada. El modelo que se propone apunta a proveer un enfoque sistémico para abordar la problemática planteada.

Palabras-clave: Financiamiento de los estudios, Estudios Universitarios, modelos de financiamiento, economía de la educación, educación chilena. 